\title{
Cylindrical shell analysis by dynamic relaxation
}

\author{
A. C. CASSELL, P. J. KINSEY \& D. J. SEFTON
}

Mrs W. L. Wood, Department of Applied Mathematics, University of Reading

In $\$ 27$ the Authors appeal for research work to be done on the methods of finding the optimum values of the time increment and damping factor in the application of dynamic relaxation to the solution of stress/strain equations. The Writer has been working on this problem and the following is a brief outline of a method of dealing with it.

30. Whether we are using two or three dimensions, the difference equations for the displacement and velocity at a particular point may be written. in partitioned matrix form as

$$
\left(\begin{array}{cc}
(2+K) \mathbf{I}_{n} & \mathbf{O}_{n} \\
\Delta t \mathbf{I}_{n} & -\mathbf{I}_{n}
\end{array}\right)\left(\begin{array}{l}
\dot{U}^{r+1} \\
\mathbf{U}^{r+1}
\end{array}\right)=\left(\begin{array}{cc}
(2-K) \mathbf{I}_{n} & \mathbf{A} \\
\mathbf{O}_{n} & -\mathbf{I}_{n}
\end{array}\right)\left(\begin{array}{l}
\dot{U}^{r} \\
\mathbf{U}^{r}
\end{array}\right) \ldots .
$$

where $\mathbf{O}_{n}$ is the $n \times n$ null matrix, $n$ being the number of dimensions,

$\mathbf{I}_{n}$ is the $n \times n$ identity matrix,

$\mathbf{U}$ is the displacement vector, e.g. in three dimensions $\mathbf{U}$ is the transpose of $(u, v, w)$,

and $\quad \mathbf{A}$ is a full $n \times n$ matrix whose coefficients are functions of the eigenvalues of the space difference operators employed.

31. Since

$$
\left(\begin{array}{cc}
(2+K) \mathbf{I}_{n} & \mathbf{O}_{n} \\
\Delta t \mathbf{I}_{n} & -\mathbf{I}_{n}
\end{array}\right)^{-1}=\left(\begin{array}{cc}
\frac{1}{(2+K)} \mathbf{I}_{n} & \mathbf{O}_{n} \\
\frac{\Delta t}{(2+K)} & -\mathbf{I}_{n}
\end{array}\right) \quad \cdots
$$

the amplification matrix for the iteration, $G$, is given by

$$
\begin{aligned}
(2+K) \mathbf{G} & =\left(\begin{array}{cc}
\mathbf{I}_{n} & \mathbf{O}_{n} \\
\Delta t \mathbf{I}_{n} & -(2+K) \mathbf{I}_{n}
\end{array}\right)\left(\begin{array}{cc}
(2-K) \mathbf{I}_{n} & \mathbf{A} \\
\mathbf{O}_{n} & -\mathbf{I}_{n}
\end{array}\right) \\
& =\left(\begin{array}{cc}
(2-K) \mathbf{I}_{n} & \mathbf{A} \\
\Delta t(2-K) \mathbf{I}_{n} & \Delta t \mathbf{A}+(2+K) \mathbf{I}_{n}
\end{array}\right)
\end{aligned}
$$

32. Hence if $\mathbf{x}$ is an eigenvector of $\mathbf{A}$ corresponding to the eigenvalue $\theta$, then

$$
\mathbf{G}\left(\begin{array}{l}
p \mathbf{x} \\
q \mathbf{x}
\end{array}\right)=\lambda\left(\begin{array}{l}
p \mathbf{x} \\
q \mathbf{x}
\end{array}\right)
$$

where $\lambda, p$ and $q$ are scalars, if

$$
\frac{(2-K) p+q \theta}{p}=\frac{(2-K) \Delta t p+q(\Delta t \theta+2+K)}{q}
$$

i.e.

$$
q^{2} \theta-p q(2 K+\theta \Delta t)-p^{2} \Delta t(2-K)=0
$$

and

$$
(2+K) \lambda=2-K+\frac{q \theta}{p}
$$

Hence, eliminating $q / p$ from equations (21) and (22),

$$
\theta=\frac{[(2+K) \lambda-2]^{2}-K^{2}}{(2+K) \Delta t \lambda}
$$

Paper published: Proc. Instn civ. Engrs, 1968, 39 (Jan.) 75-84. 
i.e. the eigenvalues $\lambda$ of the amplification matrix $\mathbf{G}$ are given by

$$
(2+K) \lambda^{2}-\lambda(4+\theta \Delta t)+2-K=0
$$

This is the generalized form of the equations $1.17,3.7$ respectively in the Writer's papers ${ }^{10,11}$ comparing dynamic relaxation with three other iterative methods and analysing its application to the solution of the biharmonic equation.

33. There is stability so long as the roots of equation (24) are complex or equal, i.e. the values of $\Delta t$ and $K$ have to be chosen so that

$$
(4+\theta \Delta t)^{2} \leqslant 4\left(4-K^{2}\right) \quad \text {. . . . . . . }
$$

for the entire range of values of $\theta$.

34. An exact analysis is possible for rectangular or box-shaped regions with boundary conditions such that the Fourier series method can be used to obtain the values of $\theta$, and suitable difference schemes. Otherwise estimates can be made. The Writer is at present working on this problem.

\section{Messrs Cassell, Kinsey and Sefton}

The Authors are grateful for Mrs Wood's contribution, which shows that the problem of numerical stability of the 'full shell' equations is still intractable. Fortunately it is possible to determine the criterion empirically by short trial runs on a computer.

36. The $n \times n$ matrix $\mathbf{A}$ of equation (15) is related to the Authors' equations (1)-(9) by $\mathbf{A}=(2[\mathbf{s}] \Delta t) / \rho h$ where [s] $\{\mathbf{u}\}$ is the finite difference matrix obtained by substituting relations (4)-(9) into equations (1)-(3).

37. It follows that the eigenvalues $\theta$ of $\mathbf{A}$ will contain $\Delta t$, and Mrs Wood's criterion (25) will need to be interpreted carefully by the engineer after he has estimated $K$. But the criterion leads to the problem of determining the full range of the eigenvalues of $(2 \Delta t / \rho h)[s]$ for the 'full shell' equations, where $n \times n$ is approximately $150 \times 150$.

38. A Paper is being prepared on an alternative approach to the numerical stability criterion leading to a simple approximate formula. However, this approach does not help with the difficult case of the 'full shell' equations.

\section{References}

10. Wood W. L. Comparison of dynamic relaxation with three other iterative methods. Engineer, 1967, 224 (5835) 683-687.

11. WooD W. L. On an explicit numerical method for solving the biharmonic equation. Numer. Math. (in preparation). 